Draft Version July 16, 2021

Typeset using LATEX twocolumn style in AASTeX61

\title{
EXPLORING THE LIMITS OF AGN FEEDBACK: BLACK HOLES AND THE STAR FORMATION HISTORIES OF LOW-MASS GALAXIES
}

\author{
I. Martín-Navarro ${ }^{1,2}$ And M. MezcuA ${ }^{3,4}$
}

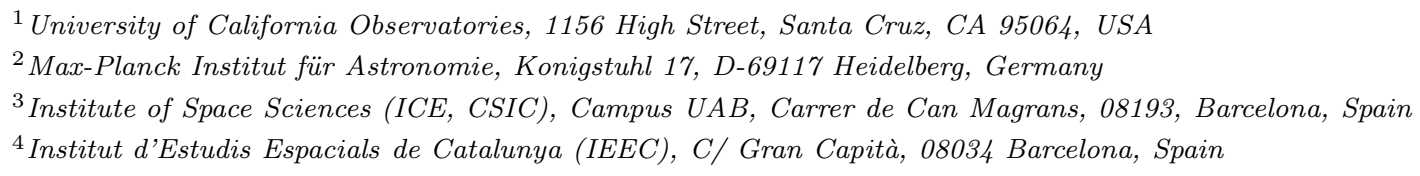

(Received; Revised; Accepted July 16, 2021)

\begin{abstract}
Energy feedback, either from active galactic nuclei (AGN) or from supernovae, is required to understand galaxy formation within a $\Lambda$-Cold Dark Matter cosmology. We study a sample of 127 low-mass galaxies, comparing their stellar populations properties to the mass of the central supermassive black hole, in order to investigate the effect of AGN feedback. We find a loose coupling between star formation history and black hole mass, which seems to suggest that AGN activity does not dominate baryonic cooling in low-mass galaxies. We also find that a break in the $M_{\bullet}-\sigma$ relation marks a transitional stellar mass, $\mathrm{M}_{\text {trans }}=3.4 \pm 2.1 \times 10^{10} \mathrm{M}_{\odot}$, remarkably similar to $\mathrm{M}_{\star}$. Our results are in agreement with a bi-modal star formation process where the AGN-dominated feedback of high-mass galaxies transitions towards a supernovae-driven regime in low-mass systems, as suggested by numerical simulations.
\end{abstract}

Keywords: galaxies: Seyfert — galaxies: active — galaxies: formation — galaxies: evolution — galaxies: star formation

Corresponding author: I. Martín-Navarro

imartinn@ucsc.edu, marmezcua.astro@gmail.com 


\section{INTRODUCTION}

The finding of tight correlations between the mass of supermassive black holes (SMBHs) at the center of massive galaxies and some of the host galaxy properties such as stellar velocity dispersion (the $M_{\bullet}-\sigma$ relation; e.g. Gebhardt et al. 2000; Ferrarese \& Merritt 2000, see Kormendy \& Ho 2013 for a review)

strongly suggest that the evolution of massive galaxies must be linked to that of their central SMBHs. Feedback from the active galactic nucleus (AGN) onto the galaxy is thought to regulate the formation of stars (and thus stellar growth) and is a necessary ingredient in cosmological simulations in order to solve the "over-cooling" problem (i.e. the too high star formation rate -SFRpredicted in the absence of feedback) and correctly reproduce the observed galaxy luminosity function, which breaks at $L_{\star}$ (e.g. Croton et al. 2006; Bower et al. 2017; Choi et al. 2017). Observational evidence for such a coupling between SMBH activity and galaxy star formation comes from the finding in galaxy clusters of largescale X-ray activities inflated by the jet of the central SMBH (e.g. Fabian et al. 2000; McNamara et al. 2000; Hlavacek-Larrondo et al. 2012) and, more recently, from the dependence of $\mathrm{BH}$ mass with SFR found for nearby massive galaxies (Martín-Navarro et al. 2016, 2018).

Low-mass galaxies, at the faint end of the luminosity function, exhibit bluer colors and have higher levels of star formation activity than red massive galaxies in which star formation is almost undetectable (e.g. Gallazzi et al. 2005; Thomas et al. 2005). In these lowmass galaxies the outflows from young stars and supernovae $(\mathrm{SNe})$ are thought to deplete the central $\mathrm{BH}$ from its gas reservoir, hampering $\mathrm{BH}$ growth and thus the impact of AGN feedback (e.g. Dubois et al. 2015; Bower et al. 2017). The transition from the AGN feedback regime that dominates massive galaxies to the $\mathrm{SN}$ feedback governing low-mass galaxies is predicted by simulations to be driven by a transition in galaxy properties at $L_{\star}$ or at a mass $M_{\star}$ of $\sim 3 \times 10^{10} \mathrm{M}_{\odot}$ and to occur already in the early Universe (e.g. Crain et al. 2015; Bower et al. 2017). Compelling observational evidence that this SN-regulated BH growth dominates over AGN feedback in dwarf galaxies is however so far lacking. The increasing number of accreting BHs being recently found in dwarf galaxies has allowed us to change this.

The finding that $\mathrm{SMBHs}$ were already in place at $\mathrm{z}>6$ suggests that they had to grow from seed BHs of $10^{2}<$ $M_{\bullet}<10^{6} \mathrm{M}_{\odot}$ in order to be able to reach a mass of more than $10^{9} \mathrm{M}_{\odot}$ in such a short time (e.g. Volonteri 2010). In the nearby Universe, such seed or intermediate-mass $\mathrm{BHs}$ (IMBHs) should be found in dwarf galaxies resembling the first galaxies formed at $\mathrm{z}>6$, with low mass and low metallicity. Optical searches for IMBHs in low-mass galaxies based on the assumption that the gas around the active $\mathrm{BH}$ is virialized has yielded several hundreds of candidates (e.g. Greene \& Ho 2004, 2007; Reines et al. 2013), in $\sim 25 \%$ of which the AGN nature has been reinforced by the detection of X-ray emission (e.g. Desroches et al. 2009; Dong et al. 2012; Baldassare et al. 2015, 2017). Direct X-ray searches have yielded the identification of a few more tens of AGN in dwarf galaxies (e.g. Reines et al. 2014; Pardo et al. 2016; Mezcua et al. 2016, 2018; see Mezcua 2017 for a review).

In this Letter we use a large sample of more than 100 low-mass galaxies with signs of AGN activity indicated by the presence of broad emission lines in their optical Sloan Digital Sky Survey (SDSS) spectrum and study any dependence of the $\mathrm{BH}$ mass with star formation activity. The absence of a clear BHSFR connection, unlike that found for massive galaxies (Martín-Navarro et al. 2018), implies that AGN feedback is not significant in these galaxies but that SN feedback must drive their growth. This is in agreement with the predictions of cosmological simulations and with the plateau observed in the low-mass regime of the $M_{\bullet}-\sigma$ correlation.

\section{SAMPLE SELECTION}

Our study includes 127 low-mass Seyfert 1 galaxies with measured stellar velocity dispersions and $\mathrm{BH}$ masses. This compilation is based on the combination of the samples presented in Xiao et al. (2011) and in Woo et al. (2015), both drawn from the SDSS. Xiao et al. (2011) based their analysis on the original sample of Greene \& Ho (2007), which selected all broadline active galaxies with $M_{\bullet}<2 \times 10^{6} \mathrm{M}_{\odot}$ in the SDSS DR4. Woo et al. (2015) used the SDSS DR7 to identify a sample of 464 narrow-line Seyfert 1 galaxies.

Stellar velocity dispersions among Xiao et al. (2011) and in Woo et al. (2015) samples were measured differently. Xiao et al. (2011) used spectroscopic data obtained with the Keck and the Clay telescopes. These observations were complemented with the velocity dispersions measurements of Barth et al. (2005). For the Woo et al. (2015) sample, stellar velocity dispersions could be directly measured from the SDSS spectra for 63 objects, combining different spectral regions in order to minimize systematics in the analysis. $\mathrm{BH}$ masses were estimated under the assumption that the gas is virialized and following the empirically estimated expression:

$$
\log \mathrm{M}_{\bullet}=\alpha+\beta\left(\frac{L_{\mathrm{H}_{\alpha}}}{10^{42} \mathrm{erg} \mathrm{s}^{-1}}\right)+\gamma\left(\frac{\mathrm{FWHM}_{\mathrm{H}_{\alpha}}}{10^{3} \mathrm{~km} \mathrm{~s}^{-1}}\right)
$$




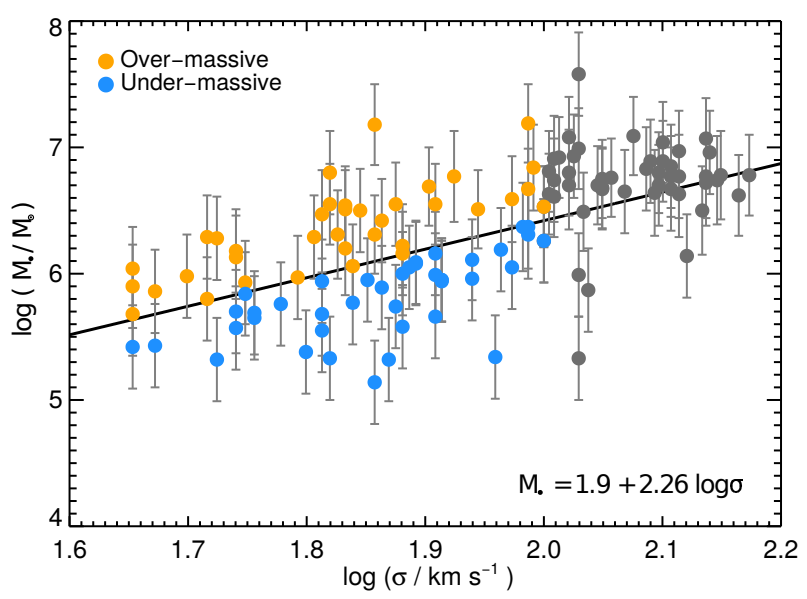

Figure 1. $M_{\bullet}-\sigma$ relation for Seyfert 1 galaxies. We restrict our stellar population analysis to over-massive (orange) and under-massive (blue) $\mathrm{BH}$ galaxies with $\sigma<100 \mathrm{~km} \mathrm{~s}^{-1}$. Black line indicates the best-fitting relation for these low- $\sigma$ galaxies. Error bars include both systematic and observational uncertainties.

where $L_{\mathrm{H}_{\alpha}}$ and $\mathrm{FWHM}_{\mathrm{H}_{\alpha}}$ are the luminosity and the full width at half maximum (FWHM) of the broadcomponent of the $\mathrm{H}_{\alpha}$ line, and $\alpha, \beta$, and $\gamma$ are calibration constants depending on modeling assumptions. Xiao et al. (2011) adopted $\alpha=6.40, \beta=0.45, \gamma=2.06$, while Woo et al. (2015) assumed $\alpha=6.544, \beta=0.46$, $\gamma=2.06$. The typical systematic uncertainty of $\mathrm{BH}$ mass measurements using this spectroscopic technique is of $\sim 0.3$ dex.

\subsection{The (local) $M_{\bullet}-\sigma$ relation}

Following Martín-Navarro et al. (2016), we investigate the interplay between $\mathrm{BH}$ activity and star formation using the $M_{\bullet}-\sigma$ relation as a metric. At a given $\sigma$, galaxies above/below the best-fitting relation have a heavier/lighter BH than the average population. Since the energy released scales up with the mass of the BH (Crain et al. 2015; Sijacki et al. 2015), galaxies with more massive BHs, e.g. above the relation, would have experienced a more intense feedback. Therefore, studying the stellar population properties across the $M_{\bullet}-\sigma$ relation probes how star formation depends on the energy input from the central BH. The universality of the $M_{\bullet}-\sigma$ relation allows us to isolate the effect of $\mathrm{BH}$ feedback.

In Fig. 1 we show the $M_{\bullet}-\sigma$ relation defined by our sample. As described above, $\mathrm{L}_{\star}$ galaxies mark a transition between SN-regulated star formation in low-mass systems, and the AGN-dominated regime of massive galaxies. Since the main motivation of this work is to constrain the effect of AGN feedback in a mass range where it is sub-dominant, we restrict our analysis to galaxies with $\sigma<100 \mathrm{~km} \mathrm{~s}^{-1}$ (colored symbols in
Fig. 1). Using the Bayesian linear regression scheme described in Kelly (2007), we find that our sample of low-mas $\left(\sigma<100 \mathrm{~km} \mathrm{~s}^{-1}\right)$ galaxies follow a best-fitting relation given by

$$
\log M_{\bullet}=1.9+2.26 \log \sigma
$$

Using this relation, shown as a solid line in Fig. 1, we sub-divide our sample into over-massive and undermassive $\mathrm{BH}$ galaxies. Over-massive $\mathrm{BH}$ galaxies are those objects above the relation (orange symbols), where the AGN effect is expected to be maximum, and undermassive $\mathrm{BH}$ galaxies (in blue) are those below the relation. The average velocity dispersion is $\sigma=69.3 \pm 2.6$ $\mathrm{km} \mathrm{s}^{-1}$ and $\sigma=73.8 \pm 2.3 \mathrm{~km} \mathrm{~s}^{-1}$ for over-massive and under-massive $\mathrm{BH}$ galaxies, respectively. This translates into a typical stellar mass of $\mathrm{M}_{\star} \sim 0.9 \times 10^{10} \mathrm{M}_{\odot}$. By construction, over-massive $\mathrm{BH}$ galaxies host heavier $\mathrm{BHs}$ than under-massive $\mathrm{BH}$ objects $\left(\log M_{\bullet}{ }^{\mathrm{OM}}=6.31 \pm 0.05\right.$ $\mathrm{M}_{\odot}$ and $\left.\log M_{\bullet}{ }^{\mathrm{UM}}=5.89 \pm 0.05 \mathrm{M}_{\odot}\right)$. Note that Eq. 2 should not be taken as an absolute reference, but rather as a local measurement. The exact behavior of the $M_{\bullet}-\sigma$ relation for low-mass galaxies and how it relates to higher-mass objects is still an open question (e.g. Wyithe 2006; Graham \& Spitler 2009; Xiao et al. 2011; Mezcua 2017), and will be discussed in detail in $\S 4$. For the purposes of this work, our best-fitting relation is fundamentally a local ruler to quantify how heavy/light a given $\mathrm{BH}$ is compared to the average population, at fixed stellar velocity dispersion.

\section{DATA AND STELLAR POPULATIONS ANALYSIS}

We have based our stellar population analysis on spectra from the SDSS DR12. Unfortunately, the low signalto-noise of the individual spectra is not high enough to obtain reliable star formation histories (SFHs). To meet the signal-to-noise requirements, we perform a Voronoi binning of the $M_{\bullet}-\sigma$ plane (Cappellari \& Copin 2003). In order to avoid confusion issues around the best-fitting relation, we first divide our sample into over-massive and under-massive $\mathrm{BH}$ galaxies, and we latter carry out the two-dimensional binning (i.e., $M_{\bullet}$ and $\sigma$ ) considering the two sub-samples separately. We impose a minimum signal-to-noise of 35 , which leads to $\sim 5$ bins within both over-massive and under-massive BH galaxies. Notice that having multiple representative spectra above and below the best-fitting $M_{\bullet}-\sigma$ relations minimizes the effect of systematics in the stellar population analysis.

After the Voronoi binning, we proceed to generate the combined spectra. We use the SDSS redshift measurements to correct for the radial velocity of each individual galaxy. We then normalize the spectra by the me- 


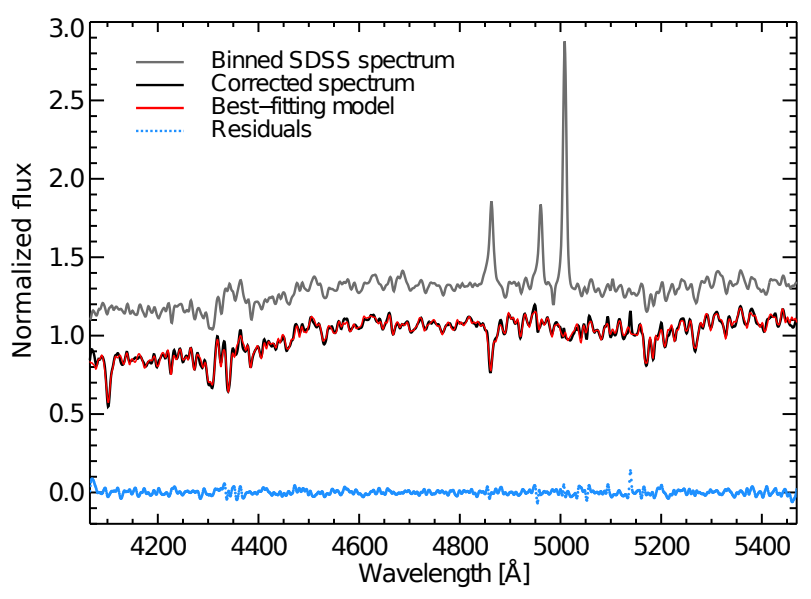

Figure 2. Spectroscopic data. We show in gray a typical Voronoi-binned SDSS spectrum, and in black the same spectrum after being corrected for non-stellar features (nebular emission plus FeII and AGN continuum). The best-fitting stellar populations model is shown in red, along with the percent-level residuals in blue.

dian flux over the $4000<\lambda<5000 \AA$ wavelength range. We also homogenize the spectral resolution of the SDSS data by smoothing the data to a common resolution of $\mathrm{FWHM}=5 \AA$, using a gaussian kernel. Although the wavelength dependence of the SDSS resolution is sometimes ignored, we make sure in this way that we do not introduce artifacts on the stellar population analysis. After applying the redshift and spectral resolution corrections, we co-add individual spectra following our Voronoi binning tessellations. In Fig. 2, the gray line shows one of these combined SDSS spectra. These are dominated by strong nebular emission lines, in addition to the Balmer $\mathrm{H}_{\delta}, \mathrm{H}_{\gamma}$, and $\mathrm{H}_{\beta}$ lines, which we subtract before attempting the stellar population analysis. To estimate the best-fitting stellar template we make use of the Penalized Pixel-Fitting (Cappellari \& Emsellem 2004, pPXF). We then use the residuals from this first fitting to individually model each emission line as the sum of two gaussians. There is no physical motivation behind a two-component fitting, but it provides a reasonably good fit to the observed spectra (see Fig. 2). Notice also that we are not interested in the properties of the emission lines.

In addition to the nebular emission, we also need to remove from our spectra the non-stellar FeII emission and the AGN continuum. This time, we feed pPXF with the FeII templates of Kovačević et al. (2010), including also a featureless (additive) continuum to model the AGN contribution. The black solid line in Fig. 2 shows one of our corrected SDSS spectra, after accounting for all the emission features (nebular + FeII + AGN continuum).

\subsection{Stellar populations}

To measure the stellar populations properties, and in particular the SFHs among our sample, we use the STEllar Content and Kinematics via Maximum A Posteriori likelihood (STECKMAP) code (Ocvirk et al. 2006). STECKMAP is a Bayesian algorithm to find the best-fitting linear combination of single stellar population models describing a given spectrum. It non-parametrically reconstructs SFHs and has been extensively tested (e.g. Sánchez-Blázquez et al. 2011; Ruiz-Lara et al. 2015). We feed STECKMAP with the MILES stellar population synthesis models (Vazdekis et al. 2010). These models cover a range in metallicity from -1.3 to +-0.22 dex, and from 67 Myr to 17 Gyr in age. We use the base MILES models, which follow the solar neighborhood abundance pattern, i.e., they are $\alpha$-enhanced $([\alpha / \mathrm{Fe}] \sim+0.4)$ for metal-poor populations, but becoming $[\alpha / \mathrm{Fe}] \sim 0.0$ for solar and super-solar metallicities. STECKMAP allows for a simultaneous analysis of the kinematical and stellar population properties. The solid red line in Fig. 2 shows the best-fitting model given the (black solid line) spectrum. Residuals (in blue) are of the order of a few percent, with no significant structure. This low level of scatter in the residuals, in particular around the prominent emission lines, suggests that the correction from AGN contamination is accurate enough for a robust stellar population analysis.

\section{RESULTS}

The average SFHs and cumulative stellar mass distributions for over-massive and under-massive (low- $\sigma$ ) galaxies are shown in Fig. 3. Shaded regions indicate the error around the mean value. The SFR as a function of look-back time shows a rather bursty behavior at all redshifts, characteristic of low-mass objects (e.g. Koleva et al. 2009).

Over-massive and under-massive $\mathrm{BH}$ galaxies in the explored $\sigma$ range exhibit rather similar SFHs. The main differences appear for very old stellar populations, where the SFR in galaxies above the best-fitting $M_{\bullet}-\sigma$ relation seem to be slightly elevated compared to those galaxies below the relation. This is reflected in the mass-weighted age, which is marginally older for overmassive than for under-massive $\mathrm{BH}$ galaxies $(5.2 \pm 0.6$ and $4.3 \pm 0.4 \mathrm{Gyr}$, respectively).

We note that the broad emission lines used in our sample as a proxy for the $\mathrm{BH}$ mass could also originate from transient stellar phases (e.g. Baldassare et al. 2016). To reinforce the AGN origin of the emission lines, we repeat the analysis but using only those objects in our sample with detected nuclear X-ray emission as a sig- 
nature of AGN activity (39 out of 127 dwarf galaxies; e.g. Desroches et al. 2009; Dong et al. 2012). The results we obtain are consistent with those found for the full sample of 127 objects.

Galaxy inclination might artificially shift the position of a given object in the $M_{\bullet}-\sigma$, as the effective velocity dispersion of a face-on rotating system becomes meaningless. Given the predominantly disky nature of our sample, this effect may significantly influence our results. We test this by homogeneously deriving the $\mathrm{b} / \mathrm{a}$ axis ratios of our sample based on the SDSS DR12 photometry. We perform then a bi-linear fitting of the $M_{\bullet} \mathbf{0}^{-}$ $\sigma$-b/a ratio plane, finding that $\log M_{\bullet}=1.8+2.28 \log$ $\sigma+0.09 \mathrm{~b} / \mathrm{a}$ and that galaxies with higher $\mathrm{b} / \mathrm{a}$ ratios tend to have lower $\sigma$. This is in agreement with the results from Xiao et al. (2011) and Woo et al. (2015) and reduces even more the differences between the SFH of the over-massive and under-massive galaxies.

As an additional test, we also create a single overmassive and under-massive $\mathrm{BH}$ galaxy spectrum by combining all the objects above and below the relation. The recovered SFHs are also fully compatible with those obtained with the binned spectra, suggesting that Fig. 3 actually reflects the average behavior of both types of galaxies and it is not driven by statistical flukes. Finally, we also investigate the effect of systematic uncertainties in the $\mathrm{BH}$ mass estimations. Considering only those objects deviating more than 0.3 dex from the $M_{\bullet}$ $\sigma$ relation, the differences in the SFHs remain similar to those shown by Fig. 3 .

\section{DISCUSSION AND CONCLUSIONS}

The rather similar SFHs of under-massive and overmassive $\mathrm{BH}$ galaxies in the low- $\sigma$ end suggest that AGN activity may not play a dominant role in regulating star formation in low-mass galaxies. Our findings offer observational support to the bi-modal paradigm of star formation within galaxies: while SN feedback is likely to regulate baryonic cooling within low-mass dark matter halos, a different, AGN-driven regime drives star formation in massive galaxies. The transition between SNand AGN-driven modes occurs at a characteristic stellar mass $\mathrm{M}_{\star} \sim 3 \times 10^{10} \mathrm{M}_{\odot}$ (e.g. Baldry et al. 2012).

To better understand this bi-modal character of star formation, we compare in Fig. 4 the SFHs of undermassive and over-massive $\mathrm{BH}$ galaxies along the $M_{\bullet}-\sigma$ relation (upper panel). For high- $\sigma$ galaxies, we show the SFHs presented in Martín-Navarro et al. (2018). BH masses in Seyfert 1 galaxies have been scaled up by +0.4 dex to match direct $\mathrm{BH}$ mass measurements. It has been pointed out that the $M_{\bullet}-\sigma$ relation flattens for low-mass galaxies, with $\mathrm{BH}$ masses asymptoti-

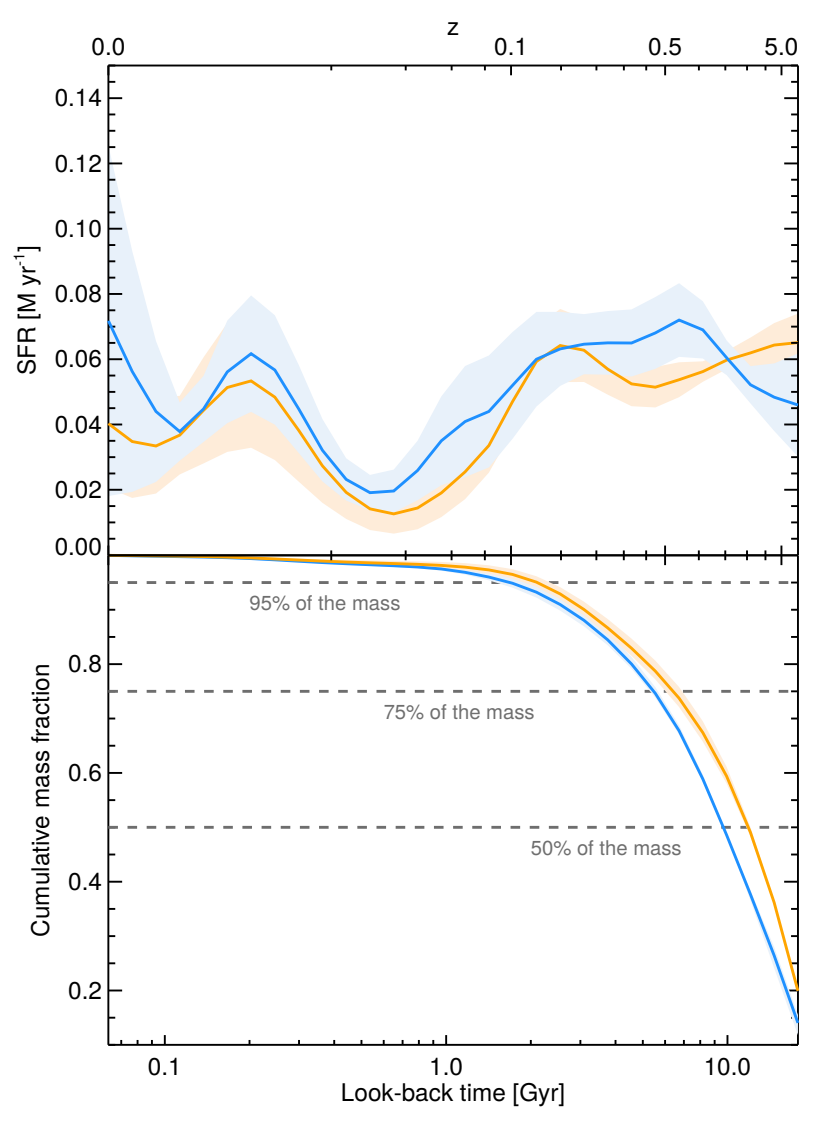

Figure 3. SFR (top) and cumulative stellar mass function (bottom) as a function of look-back time for under-massive (blue) and over-massive (orange) BH galaxies. Shaded regions correspond to the $1 \sigma$ uncertainty of the mean value. The differences between both types of objects are weak, negligible if the $\mathrm{b} / \mathrm{a}$ is taken into account. Dashed horizontal lines in the bottom panel indicate when $50 \%, 75 \%$ and $95 \%$ of the galaxy mass is reached, respectively.

cally tending towards $\log M_{\bullet} \sim 5 \mathrm{M}_{\odot}$ (Greene \& Ho 2006; Mezcua 2017), favoring a direct collapse-formation scenario (e.g. Volonteri 2010). Such a flattening is clear from Fig. 4, which combines our sample of 127 objects, plus 204 galaxies with direct BH mass measurements from van den Bosch (2016), being the largest comparison up to date. This flattening seems to suggest a weaker but still noticeable coupling between BHs and star formation, in agreement with our analysis. Our best-fitting $M_{\bullet}-\sigma$ relation for the 127 low-mass galaxies is shown in green, and that of van den Bosch (2016) is shown in red. Note that our best-fitting relation describes the average behavior of $\sigma \sim 70 \mathrm{~km} \mathrm{~s}^{-1}$ galaxies, while the sample of van den Bosch (2016) probes galaxies with $\sigma \sim 200 \mathrm{~km} \mathrm{~s}^{-1}$. Interestingly, the intersection between both fits occurs at characteristic $\sigma_{\text {trans }}=$ $96 \pm 25 \mathrm{~km} \mathrm{~s}^{-1}$, or equivalently, at a characteristic stellar mass of $\mathrm{M}_{\text {trans }}=3.4 \pm 2.1 \times 10^{10} \mathrm{M}_{\odot}($ van den Bosch 
2016), remarkably similar to $M_{\star}$. Notice that the uncertainty in $\mathrm{M}_{\text {trans }}$ is determined by the scaling factor applied to Seyfert 1 BH masses. The lower limit corresponds to uncorrected $\mathrm{BH}$ masses, and the upper limit is that shown in Fig. 4, being this the only effect of the scaling factor on our results.

We therefore hypothesize that our observations can be explained if the role of AGN feedback in regulating star formation becomes sub-dominant in low- $\sigma$ galaxies, as expected from numerical simulations (e.g. Habouzit et al. 2017). Low- $\sigma$ galaxies, with BH masses asymptotically converging towards $M_{\bullet} \sim 10^{5} \mathrm{M}_{\odot}$, would be the low-mass relics of the high- $z$ galaxy seeds. Since SN feedback is capable of preventing effective gas cooling towards the center of low- $\sigma$ galaxies (Dubois et al. 2015), the central SMBH is not able to reach the critical mass required to regulate star formation (Bower et al. 2017). The same process of black hole starvation would explain the lack of classical bulge-like morphologies in our sample (Jiang et al. 2011).

Such a scenario would also explain the flattening of the $M_{\bullet}-\sigma$ relation, as a consequence of a weaker coupling between SMBH and host galaxy. Our transition mass $M_{\text {trans }}$, equivalent to $M_{\star}$, would then separate the high-mass end regime, where star formation occurs under a hot accretion mode regulated by AGN activity, and low-mass galaxies, where SNe regulate star formation. Cold accretion would be dominant in these lowmass systems, whose central $\mathrm{BH}$ and stellar mass have not grow enough to match the standard (high-mass end) scaling relations. However, some caveats in our analysis should be noted. First, our results consist of an upper limit for the $\mathrm{BH}$ effect on baryonic cooling given the uncertainties in low- $\sigma \mathrm{BH}$ mass estimates. Second, at low galaxy stellar masses the $\mathrm{BH}$ occupation fraction is not 1 (e.g. Mezcua et al. 2018) and thus our sample may not be a complete representation of the general population. Finally, we have not directly tested whether the presence of an AGN has an impact on the star formation history of the host galaxy. Such analysis is left for when the number of low-mass galaxies with dynamical $\mathrm{BH}$ mass measurements is large enough for such study to be performed. We note though that such impact is not expected given the radically different time-scales of AGN activity and star formation.

In summary, our results suggest that the AGN effect on baryonic cooling strongly depends on galaxy mass, being weaker in low- $\sigma$ galaxies than in high- $\sigma$ objects. Deeper optical spectra of low- $\sigma$ galaxies will be needed in the future to better constrain the stellar population properties of these objects. Moreover, larger and more complete samples of the low-mass end of the galaxy mass function are needed to overcome possible systematics related to Seyfert 1 galaxies (e.g. Ricci et al. 2017).

The authors thank Pau Diaz Gallifa for his constant support. I.M.N. acknowledges funding from the Marie Skłodowska-Curie Individual Fellowship 702607, and from grant AYA2013-48226-C3-1-P from the Spanish Ministry of Economy and Competitiveness (MINECO). M.M. acknowledges support from the Spanish Juan de la Cierva program (IJCI-2015-23944).

\section{REFERENCES}

Baldassare, V. F., Reines, A. E., Gallo, E., \& Greene, J. E. 2015, ApJL, 809, L14

—. 2017, ApJ, 836, 20

Baldassare, V. F., Reines, A. E., Gallo, E., et al. 2016, ApJ, 829,57

Baldry, I. K., Driver, S. P., Loveday, J., et al. 2012, MNRAS, 421, 621

Barth, A. J., Greene, J. E., \& Ho, L. C. 2005, ApJL, 619, L151

Bower, R. G., Schaye, J., Frenk, C. S., et al. 2017, MNRAS, 465, 32

Cappellari, M., \& Copin, Y. 2003, MNRAS, 342, 345

Cappellari, M., \& Emsellem, E. 2004, PASP, 116, 138

Choi, E., Ostriker, J. P., Naab, T., et al. 2017, ApJ, 844, 31

Crain, R. A., Schaye, J., Bower, R. G., et al. 2015, MNRAS, 450, 1937
Croton, D. J., Springel, V., White, S. D. M., et al. 2006, MNRAS, 365, 11

Desroches, L.-B., Greene, J. E., \& Ho, L. C. 2009, ApJ, 698, 1515

Dong, R., Greene, J. E., \& Ho, L. C. 2012, ApJ, 761, 73

Dubois, Y., Volonteri, M., Silk, J., et al. 2015, MNRAS, 452, 1502

Fabian, A. C., Sanders, J. S., Ettori, S., et al. 2000, MNRAS, 318, L65

Ferrarese, L., \& Merritt, D. 2000, ApJL, 539, L9

Gallazzi, A., Charlot, S., Brinchmann, J., White, S. D. M., \& Tremonti, C. A. 2005, MNRAS, 362, 41

Gebhardt, K., Bender, R., Bower, G., et al. 2000, ApJL, 539, L13

Graham, A. W., \& Spitler, L. R. 2009, MNRAS, 397, 2148

Greene, J. E., \& Ho, L. C. 2004, ApJ, 610, 722

-. 2006, ApJL, 641, L21 


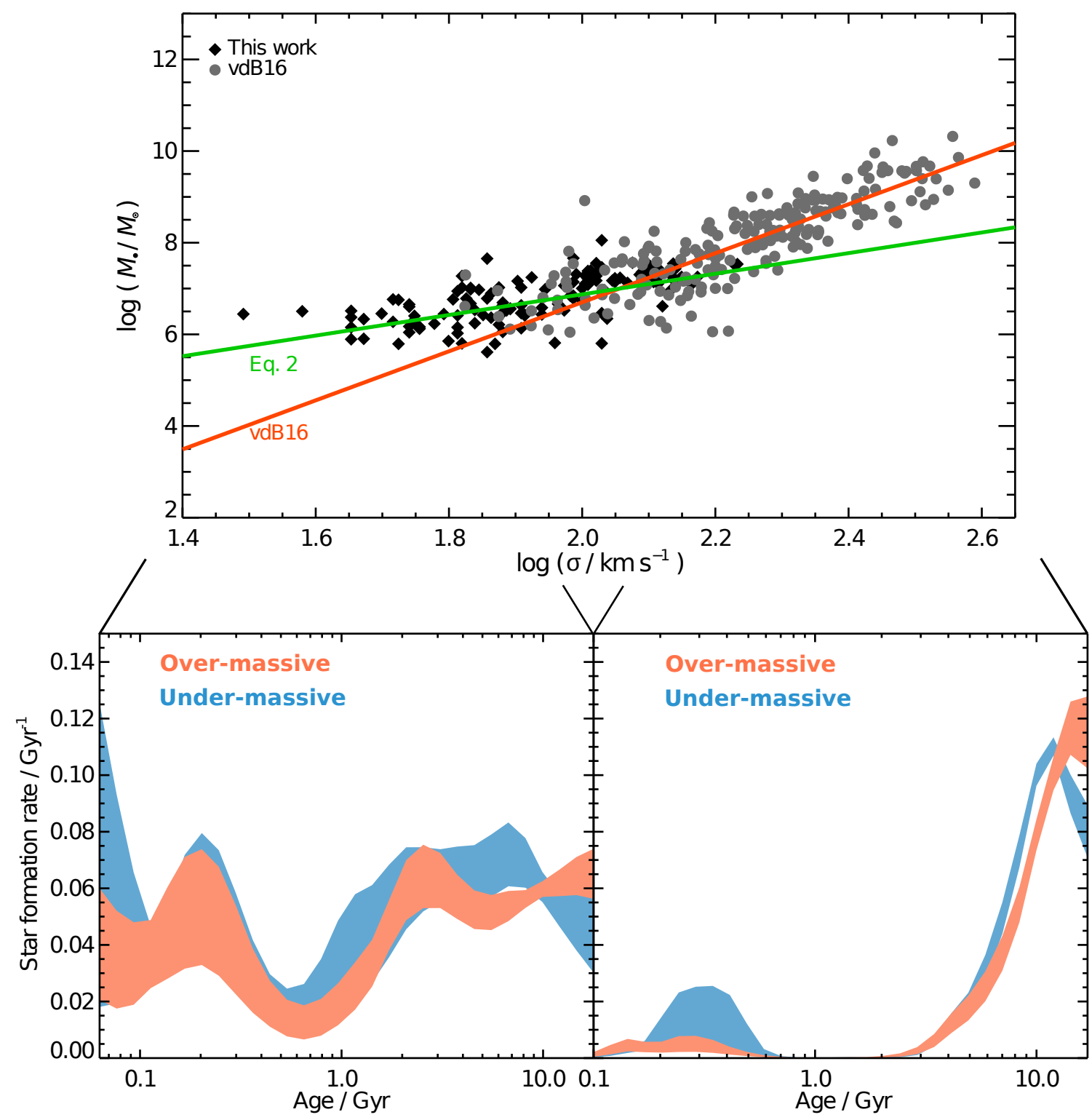

Figure 4. Upper panel shows the $M_{\bullet}-\sigma$, combining our sample (black diamonds) with that of van den Bosch (2016) for higher mass galaxies (light circles). The green line indicates our best-fitting trend for $\sigma \sim 70 \mathrm{~km} \mathrm{~s}^{-1}$ galaxies, while the red line is the relation expected for more massive object $\left(\sigma \sim 200 \mathrm{~km} \mathrm{~s}^{-1}\right)$. A characteristic $\sigma_{\text {trans }} \sim 100 \mathrm{~km} \mathrm{~s}^{-1}$ marks the transition between low-mass and high-mass scaling relations, and correspond to a stellar mass of $\mathrm{M}_{\text {trans }} \sim 3 \times 10^{10} \mathrm{M}_{\odot}$. In the bottom left and bottom right panels, we show the SFHs of over-massive (orange) and under-massive (blue) BH galaxies for low- $\sigma$ and high- $\sigma$ galaxies, respectively. We interpret the differences between these two panels as a transition process between an AGN-dominated regime for high-mass galaxies, towards a non-AGN related star formation mode for low-mass systems, probably regulated by SN feedback.

-. 2007, ApJ, 670, 92

Habouzit, M., Volonteri, M., \& Dubois, Y. 2017, MNRAS, 468, 3935

Hlavacek-Larrondo, J., Fabian, A. C., Edge, A. C., et al. 2012, MNRAS, 421, 1360

Jiang, Y.-F., Greene, J. E., Ho, L. C., Xiao, T., \& Barth, A. J. 2011, ApJ, 742, 68

Kelly, B. C. 2007, ApJ, 665, 1489
Koleva, M., de Rijcke, S., Prugniel, P., Zeilinger, W. W., \& Michielsen, D. 2009, MNRAS, 396, 2133

Kormendy, J., \& Ho, L. C. 2013, ARA\&A, 51, 511

Kovačević, J., Popović, L. Č., \& Dimitrijević, M. S. 2010, ApJS, 189, 15

Martín-Navarro, I., Brodie, J. P., Romanowsky, A. J., Ruiz-Lara, T., \& van de Ven, G. 2018, Nature, 553, 307 
Martín-Navarro, I., Brodie, J. P., van den Bosch, R. C. E., Romanowsky, A. J., \& Forbes, D. A. 2016, ApJL, 832, L11

McNamara, B. R., Wise, M., Nulsen, P. E. J., et al. 2000, ApJL, 534, L135

Mezcua, M. 2017, International Journal of Modern Physics D, 26, 1730021

Mezcua, M., Civano, F., Fabbiano, G., Miyaji, T., \& Marchesi, S. 2016, ApJ, 817, 20

Mezcua, M., Civano, F., Marchesi, S., et al. 2018, ArXiv e-prints, arXiv:1802.01567

Ocvirk, P., Pichon, C., Lançon, A., \& Thiébaut, E. 2006, MNRAS, 365, 74

Pardo, K., Goulding, A. D., Greene, J. E., et al. 2016, ApJ, 831, 203

Reines, A. E., Greene, J. E., \& Geha, M. 2013, ApJ, 775, 116

Reines, A. E., Plotkin, R. M., Russell, T. D., et al. 2014, ApJL, 787, L30
Ricci, F., La Franca, F., Marconi, A., et al. 2017, MNRAS, 471, L41

Ruiz-Lara, T., Pérez, I., Gallart, C., et al. 2015, A\&A, 583, A60

Sánchez-Blázquez, P., Ocvirk, P., Gibson, B. K., Pérez, I., \& Peletier, R. F. 2011, MNRAS, 415, 709

Sijacki, D., Vogelsberger, M., Genel, S., et al. 2015, MNRAS, 452, 575

Thomas, D., Maraston, C., Bender, R., \& Mendes de Oliveira, C. 2005, ApJ, 621, 673

van den Bosch, R. C. E. 2016, ApJ, 831, 134

Vazdekis, A., Sánchez-Blázquez, P., Falcón-Barroso, J., et al. 2010, MNRAS, 404, 1639

Volonteri, M. 2010, A\&A Rv, 18, 279

Woo, J.-H., Yoon, Y., Park, S., Park, D., \& Kim, S. C. 2015, ApJ, 801, 38

Wyithe, J. S. B. 2006, MNRAS, 365, 1082

Xiao, T., Barth, A. J., Greene, J. E., et al. 2011, ApJ, 739, 28 